\title{
User-Centred Conceptual Design of a Lower-Body Exoskeleton for Standing in Elderly
}

\author{
Ben Mound ${ }^{1}$ and Khaled M. Goher ${ }^{2}$ \\ University of Lincoln, UK, ${ }^{2} \mathrm{e}-\mathrm{mail}$ 11212026@students.lincoln.ac.uk \\ University of Lincoln, UK, ${ }^{1} \mathrm{e}$-mail: kgoher@lincoln.ac.uk
}

\begin{abstract}
Technology continues to extend the human life span with each new generation but fails to adequately support the daily challenges that come with old age. An increasing amount of elderly people experience difficulty performing daily tasks as simple as standing up from a chair causing a decrease in mental and physical wellbeing. Depending on users' perspectives, this paper designs an active lower limb exoskeleton (LLE) to support the sit to stand (STS) manoeuvre by employing multiple modelling techniques to determine the torque output required to provide $15 \%$ assistance to the wearer. The chassis of the exoskeleton is analysed using finite element analysis (FEA) software and is determined to experience acceptable amounts of stress and deformation.
\end{abstract}

\section{Introduction}

Exoskeletons are wearable devices that fix to a user's body and enhances movement or strength through amplification and increases endurance [1]. They achieve this by augmenting the wearer's inherent capabilities or by taking full bodily control. They are successfully used to facilitate rehabilitation after injury and illness and have shown promising advances in reducing strain on labourers and soldiers, allowing them to carry more load and walk further [2]. Many full and lower limb exoskeletons exist in different design and research phases but only a few have been certified for sale to consumers. Even fewer are within the normal financial capacity of those requiring them, often only being within the budget of large rehabilitation facilities. There are two main classifications of exoskeletons, passive and active. An active suit is a powered device that uses batteries or other power sources to power sensors, actuators and a computer for control [3]. Passive suits are purely mechanical and utilise springs, dampers and locking mechanisms to capture energy or redistribute load [4]. Additionally, there is the classification of a full or partial exoskeleton, a full suit enhances the movement or strength of the whole-body including arms, back and legs while partial suits cover only a targeted area or joint.

The intent of this research is to design and develop an active lower limb exoskeleton (LLE) that is lightweight, durable and easy to use by the elderly and disabled. State of the art LLE's often offer a range of functionality including, gait assist, gait rehabilitation and sit to stand (STS) manoeuvres. The scope of this project, however, is aimed at developing an LLE capable of performing the latter. A sit to stand manoeuvre is the physical transition from a seated position to a standing one, the need for such a device stems from an increasing ageing population whose independence is at risk, often relying on the constant help of carers or family members which can be a time consuming and expensive situation. Increasing a person's independence through mobility has been shown 
to have a positive impact on that persons wellbeing [5][6] and is the main drive behind this project.

\section{A. State of the Art}

The first suit was developed in 1890 by Nicholas Yagn; a rudimentary design composed of bowed springs that ran the length of the wearer's legs [7]. This enabled energy to be transferred from a spring to the user while walking and running; putting less strain on the users [1]. Due to the constraints of technology at the time very little progress had been made until the mid-1960's when General Electric started development on their full body, electro-mechanical suit called Hardiman in order to augment the users' strength allowing them to lift $680 \mathrm{Kg}$ [8]. The suit consisted of a complicated network of hydraulic actuators and electrical systems that would control both arms and pincer like hands. This designs had limited stability [8 and 9]. The first medically assistive suit was designed in the 1960's at the Mihailo Pupin institute, Serbia for rehabilitation of paraplegics and people with similar disabilities. This project led to the first active walking exoskeleton in 1970 and was an innovation of the time, using electric motors and an electronically programmed computer allowing for a smooth gait. It also drastically decreased the weight of past suits, having a lightweight frame that weighed $12 \mathrm{Kg}$ [10]. Modern exoskeletons such as Hybrid Assistive Limb (HAL), a gait assistive suit, have advanced to become smart machines carrying their own microcontrollers and on-board power supply while only being $14 \mathrm{Kg}$ in total. It utilises a sophisticated control system to sense the users' intended motion.

\section{Sit TO STAND TRANSITION}

The STS transition is a regular movement most people experience on a daily basis without difficulty, however for some, such as the elderly and disabled it can pose as a real challenge often requiring additional support, reducing their ability to stay mobile. STS manoeuvre is an increasing area of study yet there is no widely accepted standardization of the transitional stages. Etnyre and Thomas [28] attempted to reconcile this by observing force plate data produced by a sample of people with an average age of 21.8 years, utilizing a switch to detect when seat off occurred and analytical methods to define key transition phases. They suggest the STS manoeuvre consists of six phases; Initiation, counter, seat off, vertical peak, rebound and standing (Figure 1). Each phase requires a changing composition of the joints and controlled force output to shift the body's centre of mass thus completing the transition [29]. Identifying these phases provides an effect basis to compare and describe data. In [30], a healthy young person can complete this manoeuvre in 1.31 seconds and other studies have shown that the elderly often take longer at 1.82 seconds [31]. It can be seen that an actuator chosen for the design will require a quick response time to be fit this quick transition. 


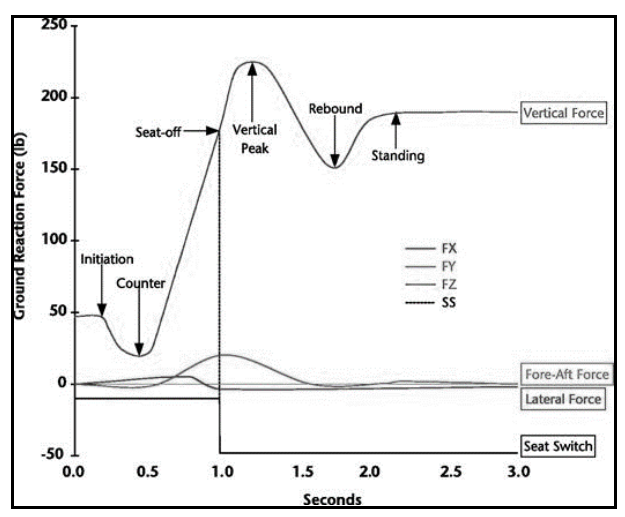

Fig1. Ground reaction forces during a sit to stand transition with the six phases identified [28]

The magnitude of the torques experienced at the hips, knees and ankles during the STS transition are the subject of much research resulting in various methods and assumptions to gain accurate results. The torques can only be estimated and not directly measured. Pala and Pratihar [32] simulated the joint torques of an able-bodied person using the simulation software ADAMS. They suggested the torque experienced at the joints are $70.22 \mathrm{Nm}$ at the ankle, $82.6 \mathrm{Nm}$ at the knee and $79 \mathrm{Nm}$ at the hip. Yoshioka et al. [33] used an inverse dynamics approach to find the joint torques of the lower limbs to validate their findings of the forces experienced in the muscles of the lower body. They found that the knee experiences a peak torque of $\sim 80 \mathrm{Nm}$ while the hip peaks at $\sim 32 \mathrm{~N}$. The ankle joint experienced a peak torque of $\sim 3 \mathrm{Nm}$ at the standing phase. Mak et al. [34] also used inverse dynamics and modelling software to find the normalised joint torques for a sample of 6 able bodied people with an average weight of $59.2 \mathrm{Kg}$. The results suggest a peak torque of $69.3 \mathrm{Nm}$ for the knee, $54.1 \mathrm{Nm}$ for the hip and $37.8 \mathrm{Nm}$ for the ankle. The aforementioned studies resulted in different torque values at the various joints with the ankle and hip joint producing the most diverse results. This may be due to the differences in the model's construction and assumptions as well as the degrees of freedom assigned to each joint when applying inverse dynamics [35].

\section{NEEDS AND EXPECTATIONS}

In this research, two surveys are created for different populations with the objective to gather qualitative data on whether potential users would find the device useful as well as quantitative data to use as design constraints. The first survey is developed for health care professionals (HCP) and the other for the general population (GP) with an interest in the older generations. Fifteen questionnaires are collected in total; four from the HCP and eleven from GPs. The HCP survey included responses from student nurses and care home managers. The GP survey included responses from care home residents and members of the public. The two surveys are conducted through telephone 
interviews, social media, focus groups and interviews. The surveys contained a brief outline of the project objective and what an exoskeleton was, including pictures. Care is taken to make the language used as easy to understand as possible with large text to facilitate the older respondents. The GP survey consisted of 10 questions while the HCP survey had eleven. Most questions are shared among both surveys with some specific for each demographic. The age range of the GP was 20-80+ with $9 \%$ of the respondents being 10-19, 9\% 30-39, $9 \%$ 40-49 and 9\% 70-79. 18\% were 50-59 and 18\% 60-69 while $27 \%$ were aged $80+$ and $36 \%$. Both demographics are asked to pick three of six options on what they considered to be the most important features of an LLE. Safety was not included as this was considered top priority by default. It can be seen in Figure 2 that both groups responded heavily to the ease of use and comfortability while the GP responded highly to lightweight and low cost. This is understandable as being light weight would allow them to fit the device themselves and remain more independent while low cost enables them more opportunity to purchase it. HCP was the only group to consider slim design an important feature, this is due to the environment both groups imagined the device being used in, it would be understandable that the HCP envision the device in a medical environment where space is limited while the GP imagined using the device at home where perhaps this is not as much an issue.

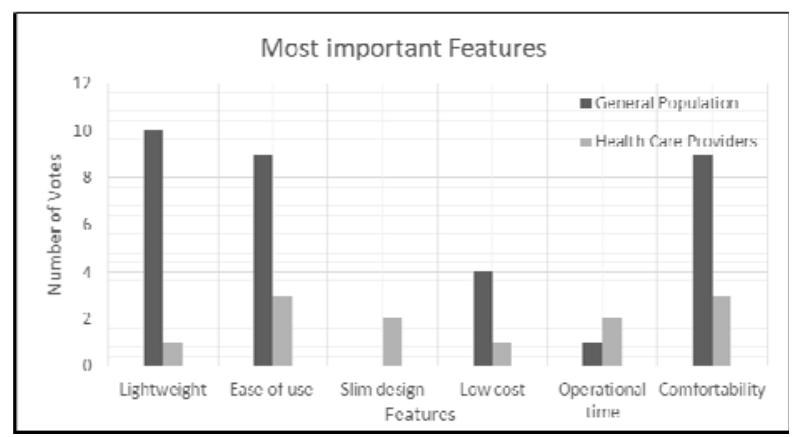

Fig. 2 Most important features" survey results

Another question posed to both groups was "Do you have any concerns about the device? This allowed a more open-ended question that could produce unexpected answers. Interestingly there was quite a divide of opinions between the HCP and GP with only two areas of concern shared, being self-fitting; receiving the highest concern with $23 \%$ and safety with $12 \%$, see Figure 3 . Other areas of concern include; reliability and softness at $12 \%$ and complexity, stability, durability, bulkiness, appropriate training, and maintenance of the device at $9 \%$. 


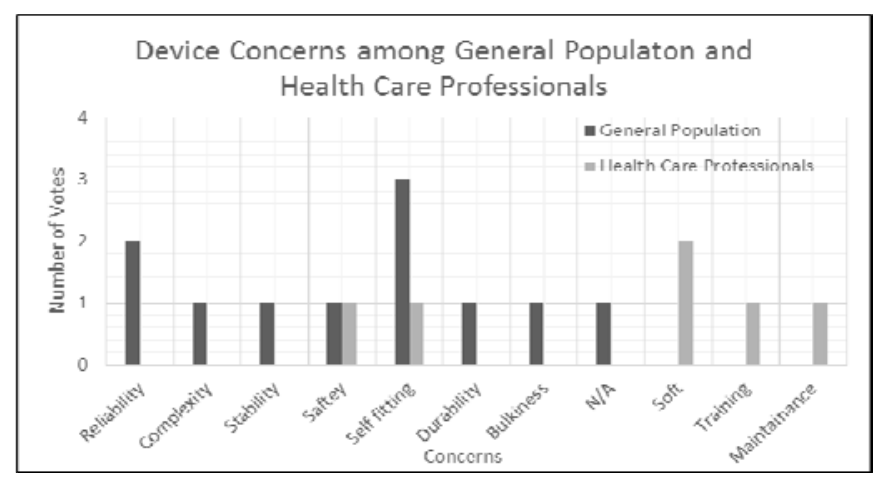

Fig.3 "Do you have any concerns about the device" survey results

A. House of Quality

III. SYSTEM DESIGN PROCESS

Data collected from the survey results informed the design process which started with decision matrix. A house of quality using Quality Functional Deployment (QFD) is used to translate the customer requirements to engineering metrics and identify key design points. The technique associates customer requirements to engineering metrics with a weighted value based on the research collected and by recognising the importance of their relationships. The QFD analysis determined that the number of steps to activate the device was the most important design point with a weight/importance of 285.7 followed by battery life with 248.6. Component price was third with 234.3 and is linked with the majority of the customer needs and so is a particularly important design point. Maximum stress came in fourth with 228.6 and is strongly linked with safety and durability and will therefore also be considered a key design point.

\section{B. System Model and FEA}

A concept design is initiated with the stages of the STS transition appears in Figure 4. A finite element analysis (FEA) using ANSYS software is used to analyse the stress, deformation and safety factor of the LLE to determine if the material chosen was suitable for the forces experienced in the chassis. This was achieved by applying the forces and boundary conditions to the geometry of the model. The geometry was imported into a static structural simulation as .step files where each part was meshed and assigned a joint. 15\% of the body segment weights and the component weights were added at the centre of masses (COMs) found previously and a moment load is applied at the hip and knee joint with the maximum motor torques found from the dynamic analysis; $19 \mathrm{Nm}$ for the hip and $32.4 \mathrm{Nm}$ for the knee. The LLE is positioned in the orientation these torques are experienced and the structure is considered fixed to the floor (Figure 5). 


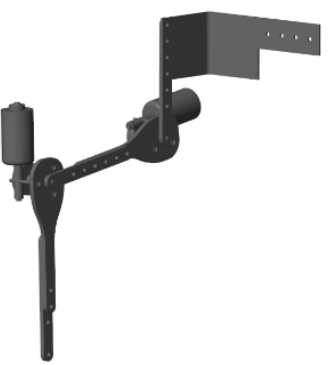

(a) Initiation

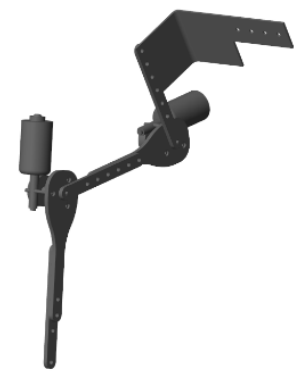

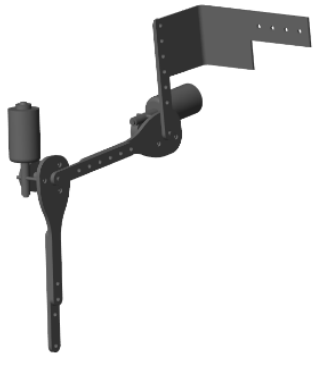

(b) Counter

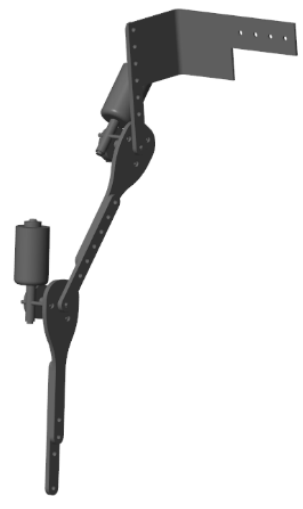

(e) Rebound

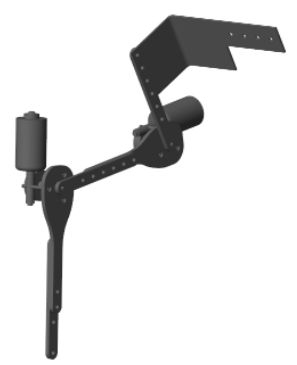

(c) Seat Off

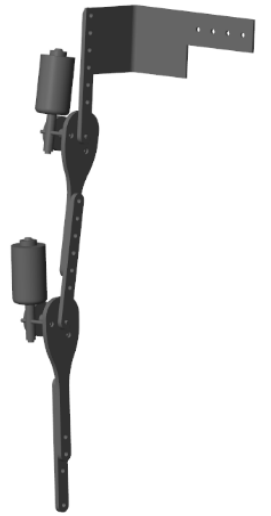

(f) Standing

(d) Vertical Peak

Fig.4 Stages of standing of the design concept

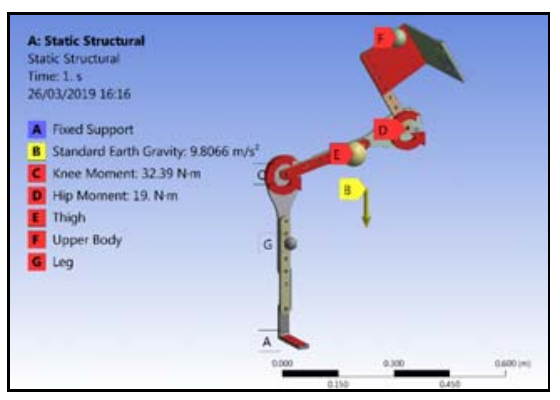

Fig. 5 FEA model with forces and boundary conditions

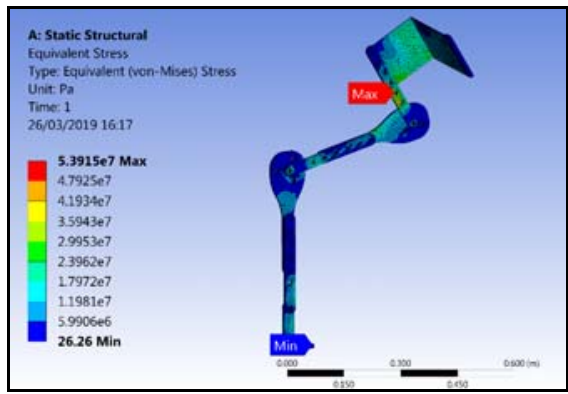

Fig. 6 FEA stress analysis of 
The maximum stress experienced in the design was found in the top extension bar around the second bolt hole with a magnitude of $53.915 \mathrm{MPa}$ as seen in Figure 6. Stress is seen in the middle extension bar also culminating around the bolt holes, but this is of a much lower magnitude. The top shank experiences a maximum values of the stress. About its curved surface but due to the shape; stress risers are avoided. Some stress is seen at the shaft holes of the knee joint and at the hip joint. Below the knee joint experiences relatively little stress. Deformation of the material is an important factor to the design as any warping may cause surfaces to rub, creating friction and stress points as well as failure. The maximum deformation is seen in the top shank with a magnitude of 0.0025633m (Figure 27). This is believed to be within a reasonable limit.

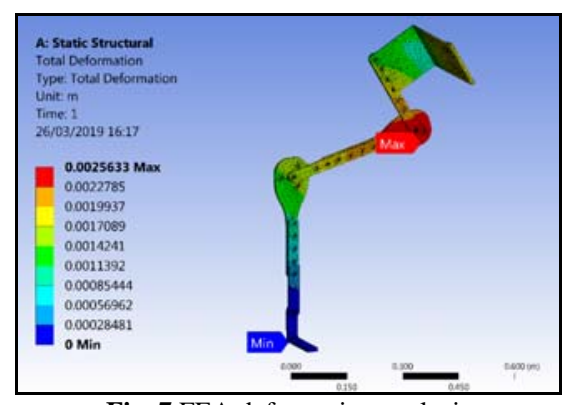

Fig. 7 FEA deformation analysis

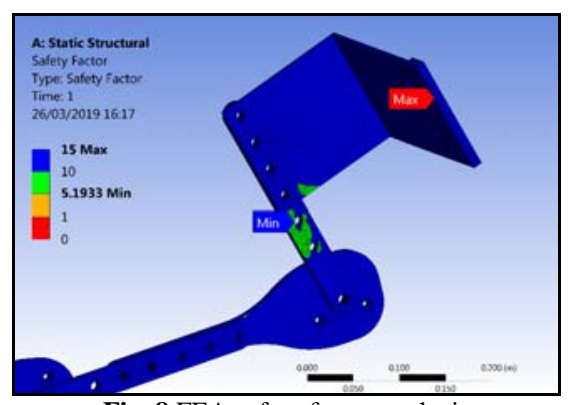

Fig. 8 FEA safety factor analysis

The safety factor is an important tool in structural analysis as it provides an estimation of failure presented as a numerical value based on the ratio of material strength over stress. A value less than one indicates that the stress experienced by the material has exceeded its strength and has therefore failed. A minimum factor of safety is often employed to limit the likelihood of failure. The minimum safety factor presented in the LLE is 5.1933 found in the top extension bar (Figure 8), this considered a good result and suggests the likelihood of failure is very low.

\section{CONCLUSION}

This paper presented a concept of designing a lower-body exoskeleton for standing in elderly people. The design process of the device started with identifying the needs and expectations using surveys to gather qualitative data on whether potential users would find the device useful as well as quantitative data to inform design process of the device. Lightweight, ease of use and being comfortable are found to be the top features as per the collected data from surveys. Reliability, self-fitting and being soft are the main concern priorities from the device from respondents' perspectives. FEA analysis of the developed concept has been investigated ahead of proceeding to the detailed design process. In brief, it is important to understand and analyse end users' needs and expectations before staring any design process. 


\section{REFERENCES}

[1] H. Ali, "Bionic Exoskeleton: History, Development and the Future.”

[2] M. Keller, "Do You Even Lift, Bro? Hardiman Was GE's Muscular Take On The HumanMachine Interface - GE Reports,” 2016. [Online]. Available: https://www.ge.com/reports/do-you-even-lift-bro-hardiman-and-the-human-machineinterface/. [Accessed: 16-Oct-2018].

[3] T. Rahman et al., "Passive exoskeletons for assisting limb movement.,” J. Rehabil. Res. Dev., vol. 43, no. 5, pp. 583-90.

[4] Economic and social research council, "Independence crucial to wellbeing in older age," 2011. [Online]. Available: https://esrc.ukri.org/news-events-and-publications/evidencebriefings/independence-crucial-to-wellbeing-in-older-age/. [Accessed: 16-Oct-2018].

[5] A. For and F. Walking, “No. 420,179,” no. 420, Jan. 1890.

[6] T. Kellner, “The Story Behind The Real 'Iron Man' Suit,” GE Reports, 2010. [Online]. Available: https://www.ge.com/reports/post/78574114995/the-story-behind-the-real-ironman-suit/. [Accessed: 16-Oct-2018].

[7] R. Bogue, "Exoskeletons and robotic prosthetics: a review of recent developments," Ind. Robot An Int. J., vol. 36, no. 5, pp. 421-427, Aug. 2009.

[8] Mihailo Pupin Institute, "History - Mihailo Pupin Institute - Robotics Laboratory." [Online]. Available: http://www.pupin.rs/RnDProfile/history.html. [Accessed: 16-Oct2018].

[9] Cyberdyne, "HAL for Well-Being Lower Limb Type Pro - CYBERDYNE.” [Online]. Available: https://www.cyberdyne.jp/english/products/fl05.html. [Accessed: 16-Oct-2018].

[10] A. Wall, J. Borg, and S. Palmcrantz, "Clinical application of the Hybrid Assistive Limb (HAL) for gait training ”a systematic review,” Front. Syst. Neurosci., vol. 9, p. 48, Mar. 2015.

[11] Office Of National Statistics, "Overview of the UK population - Office for National Statistics,” $2017 . \quad$ [Online]. Available: https://www.ons.gov.uk/peoplepopulationandcommunity/populationandmigration/populati onestimates/articles/overviewoftheukpopulation/july2017. [Accessed: 16-Oct-2018].

[12] A. J. Young, H. Gannon, and D. P. Ferris, “A Biomechanical Comparison of Proportional Electromyography Control to Biological Torque Control Using a Powered Hip Exoskeleton,” Front. Bioeng. Biotechnol., vol. 5, p. 73, Jun. 2017.

[13] NHS, "Osteoarthritis - NHS," 2016. [Online]. Available: https://www.nhs.uk/conditions/osteoarthritis/. [Accessed: 16-Oct-2018].

[14] R. F. Loeser, "Age-related changes in the musculoskeletal system and the development of osteoarthritis.,” Clin. Geriatr. Med., vol. 26, no. 3, pp. 371-86, Aug. 2010.

[15] Indramat, "Linear Actuator vs. Rotary Actuator - Indramat Products.” [Online]. Available: http://www.indramat-us.com/linear-actuator-vs-rotary-actuator/. [Accessed: 22-Oct-2018].

[16] A. Ansari, G. C. Atkeson, H. Choset, and M. Travers, “A Survey of Current Exoskeletons and Their Control Architectures and Algorithms,” 2015.

[17] R. Robotics Ltd et al., "ReWalk Robotics Ltd United States Securities and Exchange Commission," 2014.

[18] B. Chen et al., "Recent developments and challenges of lower extremity exoskeletons," $J$. Orthop. Transl., vol. 5, pp. 26-37, Apr. 2016.

[19] P. Stewart, "Pentagon looks to exoskeletons to build 'super-soldiers' | Reuters," Reuters, 2018. [Online]. Available: https://www.reuters.com/article/us-usa-militaryexoskeleton/pentagon-looks-to-exoskeletons-to-build-super-soldiers-idUSKCN1NY2Y4. [Accessed: 01-Feb-2019].

[20] A. B. Zoss, H. Kazerooni, and A. Chu, "Biomechanical Design of the Berkeley Lower Extremity Exoskeleton (BLEEX),” Trans. MECHATRONICS, vol. 11, no. 2, 2006.

[21] A. Ansari, C. G. Atkeson, H. Choset, and M. Travers, "A Survey of Current Exoskeletons and Their Control Architectures and Algorithms (Draft 4.0),” 2015.

[22] P. Komdeur, F. E. Pollo, and R. W. Jackson, "Dynamic knee motion in anterior cruciate impairment: a report and case study.,” Proc. (Bayl. Univ. Med. Cent)., vol. 15, no. 3, pp. 257-9, Jul. 2002.

[23] A. Sedkyadly and M. B. Abdelhalim, “Analyzing and Measuring Human Joints 
Movements using a Computer Vision System,” 2012.

[24] I. P. Herman, Physics of the human body. Springer, 2007.

[25] B. Etnyre and D. Q. Thomas, "Event Standardization of Sit-to-Stand Movements,” Phys. Ther., vol. 87, no. 12, pp. 1651-1666, Dec. 2007.

[26] E. G. Frykberg and K. C. Hager, "Movement analysis of sit-to-stand - researchinforming clinical practice,” Phys. Theraoy Rev., vol. 20, no. 3, pp. 156-167, 2015.

[27] F. Mourex, T. Pozzo, I. Rouhier-Marcer, and J.-P. Didier, "A kinematic comparison between elderly and young subjects standing up from and sitting down in a chair,” 1998.

[28] F. Mourey, A. Grishin, P. d'Athis, T. Pozzo, and P. Stapley, "Standing Up From a Chair as a Dynamic Equilibrium Task: A Comparison Between Young and Elderly Subjects,” Journals Gerontol. Ser. A Biol. Sci. Med. Sci., vol. 55, no. 9, pp. B425-B431, Sep. 2000.

[29] A. R. Pal and D. K. Pratihar, "Algorithm, Estimation of Joint Torque and Power Consumption during Sit-toStand Motion of Human-being Using a Genetic,” 2016.

[30] Y. Shinsuke, A. Nagano, D. C. Hay, and S. Fukashiro, "The minimum required muscle force for a sit-to-stand task,” J. Biomech., vol. 45, no. 4, pp. 699-705, Feb. 2012.

[31] M. K. Y. Mak, O. Levin, J. Mizrahi, and C. W. Y. Hui-Chan, "Joint torques during sit-tostand in healthy subjects and people with Parkinson’s disease,” Clin. Biomech., vol. 18, no. 3, pp. 197-206, Mar. 2003.

[32] D. J. Cleather and A. M. J. Bull, "Knee and hip joint forces - sensitivity to the degrees of freedom classification at the knee,” Proc. Inst. Mech. Eng. Part H J. Eng. Med., vol. 225, no. 6, pp. 621-626, Jun. 2011.

[33] Y. R. Mao et al., "The Crucial Changes of Sit-to-Stand Phases in Subacute Stroke Survivors Identified by Movement Decomposition Analysis,” Front. Neurol., vol. 9, p. 185, Mar. 2018

[34] M. R. Fotoohabadi, E. A. Tully, and M. P. Galea, "Kinematics of Rising From a Chair," Phys. Ther., vol. 90, no. 4, pp. 561-571, Apr. 2010.

[35] K. Anam and A. A. Al-Jumaily, “Active Exoskeleton Control Systems: State of the Art,” Procedia Eng., vol. 41, pp. 988-994, 2012. 